\title{
Immune reconstitution in the sigmoid colon after long-term HIV therapy
}

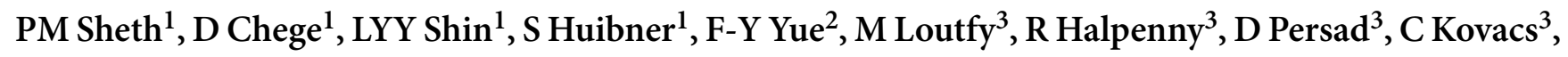 \\ T-W Chun ${ }^{4}$, G Kandel ${ }^{5}$, M Ostrowski ${ }^{2}$ and R Kaul ${ }^{1,6}$
}

Early and profound CD4 + T-cell depletion in gut-associated lymphoid tissue (GALT) may drive Human Immunodeficiency Virus (HIV) immunopathogenesis, and GALT immune reconstitution on highly active antiretroviral therapy (HAART) may be suboptimal. Blood and sigmoid colon biopsies were collected from HAART-treated individuals with undetectable blood HIV RNA for $\geqslant 4$ years and from uninfected controls. HIV proviral levels and T-cell phenotype/function were examined in both compartments. CD4 + T-cell reconstitution in the sigmoid, including CD4 + T cells expressing CCR5, exceeded that in blood and did not differ from uninfected controls. Sigmoid HIV proviral load was not correlated with CD4 + reconsitution, but was correlated with the degree of mucosal CD8 + T-cell immune activation. Colonic Gag-specific T-cell responses were common, but were not associated with proviral load or immune activation. In this select study population, long-term HAART was associated with complete CD4 + T-cell reconstitution in sigmoid colon. However, colonic immune activation may drive ongoing HIV replication.

\section{INTRODUCTION}

Human Immunodeficiency Virus-1 (HIV) infects almost 40 million people worldwide. ${ }^{1}$ Although HIV infection is characterized by progressive immunodeficiency at a systemic level, $\mathrm{HIV}$ is usually acquired across a mucosal surface (the genital or gastrointestinal mucosa), and mucosal events are increasingly recognized as critical to HIV pathogenesis. ${ }^{2} \mathrm{CD} 4+\mathrm{T}$-cell levels in blood serve as reliable prognostic markers for HIV clinical outcome, but recent studies have shown that the most profound and early CD4 + depletion occurs in the gastrointestinal-associated lymphoid tissue (GALT), which houses up to $80 \%$ of CD4+ T cells in the body. ${ }^{3-6}$ In striking contrast to the gradual depletion of CD4 + T cells observed in peripheral blood, almost 90\% of GALT CD $4+$ T cells are depleted within 2 weeks of HIV infection, ${ }^{5,7-9}$ and it has been hypothesized that these mucosal events may pave the way for progressive immune dysfunction and death. ${ }^{10}$

Highly active antiretroviral therapy (HAART) rapidly reduces the level of plasma HIV viremia and is accompanied by a more gradual reconstitution of blood CD4 + T-cell populations and dramatic improvements in life expectancy. ${ }^{11}$ Prior studies have suggested that CD4 + T-cell depletion in the gastrointestinal tract may not be reversed to the same degree as in blood. ${ }^{12,13}$ Although such studies generally evaluated the impact of relatively short-term HAART ( $<4$ years), ${ }^{12,13}$ recent work demonstrated incomplete reconstitution in the GALT of the small intestine even after prolonged, effective therapy. ${ }^{14}$ This suggests either that immunologic reconstitution in the gastrointestinal tract takes longer than in blood or that HAART is simply unable to reconstitute gastrointestinal CD4 + T-cell populations. As depletion of gastrointestinal CD $4+\mathrm{T}$ cells has been associated with reduced expression of genes important for digestive functions, reduced nutrient absorption, and increased mucosal expression of genes associated with inflammation/immune activation, this may have important clinical consequences. ${ }^{15,16}$

$\mathrm{HIV}$ infection is associated with increased immune activation in the GALT. ${ }^{17}$ Systemic immune activation has been associated with a higher HIV blood viral load and faster disease progression to acquired immune deficiency syndrome. ${ }^{18-20}$ Regulatory T cells (CD4 + CD25 + ) that suppress immune activation have been associated with lower HIV viremia and higher peripheral CD4/CD8 T-cell ratios. ${ }^{21,22} \mathrm{HIV}$-specific cellular

\footnotetext{
${ }^{1}$ Department of Medicine, University of Toronto, Toronto, Ontario, Canada. ${ }^{2}$ Department of Immunology, University of Toronto, Toronto, Ontario, Canada. ${ }^{3}$ Canadian Immunodeficiency Research Collaborative, Toronto, Ontario, Canada. ${ }^{4}$ Laboratory of Immunoregulation and Office of Clinical Research, National Institute of Allergy and Infectious Diseases, National Institutes of Health, Bethesda, Maryland, USA. ${ }^{5}$ Division of Gastroenterology, St Michael's Hospital, Toronto, Ontario, Canada. ${ }^{6}$ Department of Medicine, University Health Network, Toronto, Ontario, Canada. Correspondence: PM Sheth (prameet.sheth@utoronto.ca) 
Table 1 Characteristics of study participants

\begin{tabular}{|c|c|c|c|c|c|c|c|}
\hline No. & ID & Age (years) & $\begin{array}{c}\text { Absolute } \\
\text { blood CD4 } \\
\text { count }\left(/ \mathrm{mm}^{3}\right)\end{array}$ & $\begin{array}{c}\text { Blood HIV } \\
\text { viral load } \\
\text { (copies/ml) }\end{array}$ & $\begin{array}{c}\text { Time on } \\
\text { HAART } \\
\text { (months) }\end{array}$ & $\begin{array}{c}\text { Nadir CD4 } \\
\text { count }\left(/ \mathrm{mm}^{3}\right)\end{array}$ & $\begin{array}{l}\text { Duration of complete } \\
\text { viral suppression } \\
\text { (months) }\end{array}$ \\
\hline 1 & 003 & 47 & 540 & $<50$ & 132 & 56 & 120 \\
\hline 2 & 004 & 50 & 440 & $<50$ & 86 & 136 & 75 \\
\hline 3 & 006 & 51 & 2,260 & $<50$ & 92 & 110 & 91 \\
\hline 4 & 007 & 44 & 970 & $<50$ & 192 & 91 & 103 \\
\hline 6 & 009 & 54 & 560 & $<50$ & 180 & NA & 112 \\
\hline 7 & 010 & 59 & 730 & $<50$ & 110 & NA & 107 \\
\hline 8 & 011 & 55 & 970 & $<50$ & 120 & 82 & 115 \\
\hline 9 & 012 & 50 & 830 & $<50$ & 92 & 156 & 73 \\
\hline 13 & 018 & 43 & 580 & $<50$ & 102 & NA & 70 \\
\hline 14 & 020 & 61 & 590 & $<50$ & 85 & NA & 69 \\
\hline 15 & 021 & 62 & 420 & $<50$ & 105 & NA & 115 \\
\hline 16 & 022 & 27 & 780 & $<50$ & 51 & NA & 49 \\
\hline 17 & 037 & 41 & 810 & $<50$ & 84 & 260 & 76 \\
\hline 18 & 173 & 38 & 640 & $<50$ & 61 & 240 & 49 \\
\hline 19 & 211 & 43 & 530 & $<50$ & 175 & 170 & 56 \\
\hline 20 & 222 & 38 & 950 & $<50$ & 59 & 308 & 57 \\
\hline 21 & 249 & 50 & 1,030 & $<50$ & 141 & NA & 130 \\
\hline
\end{tabular}

Abbreviations: HAART, highly active antiretroviral therapy; HIV, Human Immunodeficiency Virus; NA, not applicable.

aThe sensitivity of viral load assays varied over time, from a threshold of >10,000 RNA copies/ml (1995) to >50 RNA copies/ml (currently).

immune responses also play an important role in host immune control. $^{23,24}$ Specifically, CD8 + T-cell immune responses targeting HIV Gag have been associated with lower HIV RNA plasma viral load and delayed disease progression to acquired immune deficiency syndrome. ${ }^{24,25}$ Strong HIV-specific CD8+ $\mathrm{T}$-cell responses may be present in the gastrointestinal mucosa of HIV-infected individuals, ${ }^{26}$ although their role in local (mucosal) HIV immune control and/or immunopathogenesis is not clear.

We hypothesized that, due to the absence of local HIV replication, long-term suppressive HAART would be associated with complete immune reconstitution in the sigmoid colon and a loss of HIV-specific CD8 + T-cell responses in both the blood and the colonic mucosa. To address this hypothesis, we have examined the phenotype and function of T cells from the blood and sigmoid colon of HIV-infected men on long-term, completely suppressive HAART.

\section{RESULTS}

\section{Study participants}

The study population consisted of $23 \mathrm{HIV}$-infected men on long-term HAART and three HIV-uninfected controls
(Table 1). The mean duration of prior HAART was 120 months (range, 51-216 months), and the mean duration of viral suppression below the level of detection was 89 months (range, 49-130 months). Therapy had been initiated for a mean of 28 months (range, 2-108 months) after the first HIV-positive enzymelinked immunosorbent assay. For those participants with data available, the mean nadir CD $4+$ T-cell count had been $183 / \mathrm{mm}^{3}$ (range, $32-360 / \mathrm{mm}^{3}$ ).

\section{CD4 + T-cell populations in the blood and the sigmoid colon}

The proportion of T cells expressing CD $4+$ in the sigmoid colon of highly supressed participants was significantly higher than that in the peripheral blood (mean, 56 vs. $45 \%$, respectively; paired $t$-test $P=0.001$; Figure $1 \mathbf{a}$ and $\mathbf{b}$ ) and was similar to that seen in HIV-uninfected controls (mean, 56 vs. $64 \%$; $P=0.14$; Figure 1a and b). However, blood CD $4+\mathrm{T}$-cell proportions in blood remained lower in HIV-infected participants (45 vs. 65\% respectively, $P=0.008$; Figure 1a).

Earlier studies described incomplete reconstitution of CD4+ $\mathrm{T}$ cells coexpressing CCR $5+$ in the recto-sigmoid despite HAART. ${ }^{13}$ CCR5 + expression was assayed in 10 out of 23 (43\%) HIV-infected participants on long-term HAART, with similar 


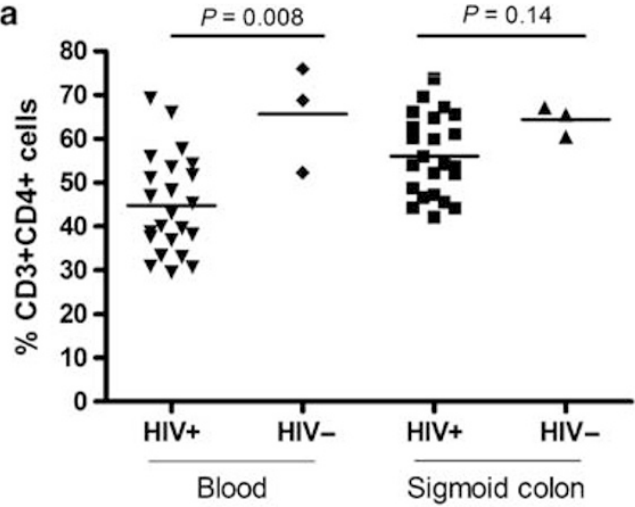

b
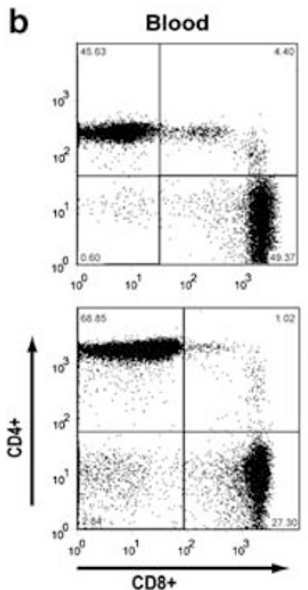
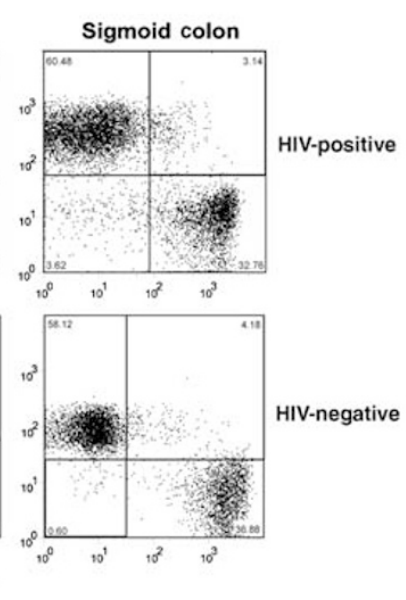

C

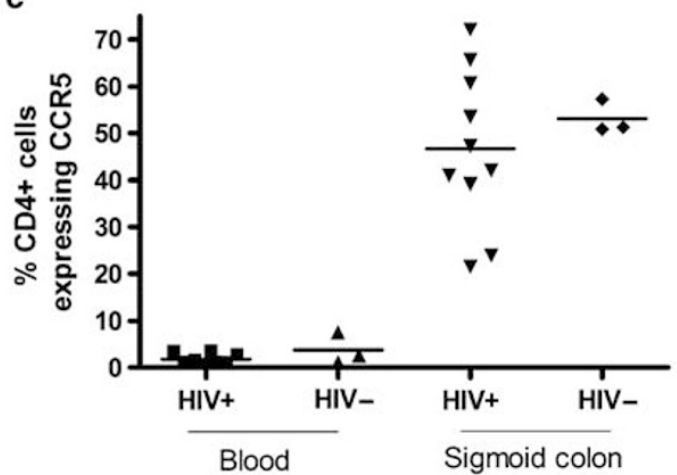

d

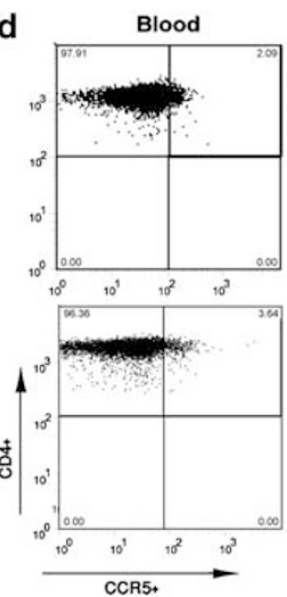

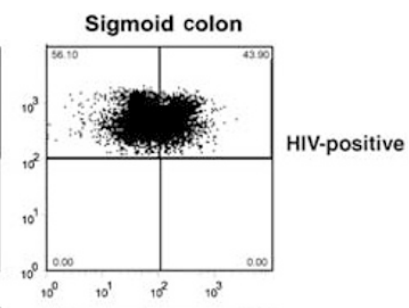

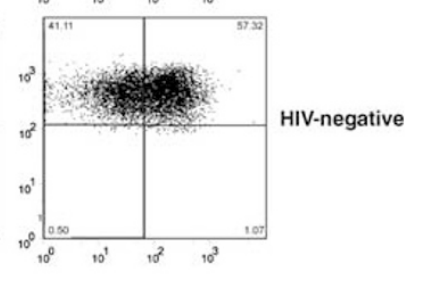

Figure $1 \mathrm{~T}$-cell proportions in blood and sigmoid colon. (a) CD4 + T-cell populations were similar in the sigmoid colon, but not in the blood, of highly suppressed and uninfected individuals. (b) Representative dot plot of CD4/CD8 proportions in blood (left panels) and sigmoid colon (right panels) of HIV-infected individuals on long-term HAART (upper panels) and HIV-negative controls (lower panels). (c) Proportion of CD4+ T cells coexpressing CCR5 in HIV-infected individuals on long-term HAART in blood and sigmoid colon. (d) Representative dot plots comparing CD4+CCR5+ coexpression in HIV-infected (upper panels) and HIV-negative controls (lower panels) in blood (left panels) and sigmoid colon (right panels). HAART, highly active antiretroviral therapy; HIV, Human Immunodeficiency Virus.

levels as in HIV-negative controls seen in both blood (mean $\%$ CD $4+$ CCR $5+, 1.8$ vs. $3.8 \%$ respectively; $P=0.15$; Figure 1c and d) and sigmoid colon (mean \%CD4 + CCR5 +, 47 vs. $53 \%$ respectively, $P=0.53$; Figure $1 \mathbf{c}$ and $\mathbf{d}$ ).

Participants had been on HAART for different lengths of time (range, 51-216 months), and the duration of complete viral suppression also varied (range, 49-130 months). The prior duration of HAART did not correlate with CD4 + T-cell reconstitution in blood $(r=0.16 ; P=0.50)$ or sigmoid colon $(r=0.14 ; P=0.55)$, although those treated for $>10$ years tended to have higher sigmoid CD4 + T-cell proportions than those treated for $<5$ years (58 vs. $50 \%$, respectively; $P=0.065$ ). Although the duration of undetectable viral load in blood correlated with improved blood CD4 + T-cell reconstitution $(r=0.62 ; P=0.002)$, this was not the case for sigmoid $(r=0.18 ; P=0.6)$. Pretherapy nadir $\mathrm{CD} 4+$ $\mathrm{T}$-cell counts did not correlate with immune reconstitution at either site (data not shown).

\section{Associations of HIV proviral load in CD4 + T cells in blood and sigmoid colon}

HIV RNA was undetectable in blood of all HIV-infected individuals, and was not measured in sigmoid colon. HIV proviral
DNA was assayed in the blood of all participants and in the sigmoid colon of 11 out of 23 individuals (48\%). Although there was a negative correlation between proviral HIV DNA levels and CD4 + T-cell proportions in blood $(r=-0.41 ; P=0.04$; Figure 2a), no such correlation was observed in the sigmoid colon $(r=0.31 ; P=0.39)$. As CD4 + T-cell populations in blood and sigmoid were isolated using different techniques, we were unable to directly compare proviral HIV DNA levels between these two compartments.

Expression of the early activation marker CD69 was much higher in colonic than in peripheral blood $\mathrm{T}$ cells, as expected, on both CD $4+\mathrm{T}$ cells ( 40.7 vs. $0.3 \% ; P<0.001$; Figure $2 \mathbf{b}$ ) and CD8 + T cells ( 35.4 vs. $0.7 \% ; P<0.001$; Figure $2 \mathbf{b}$ ). The same applied to expression of HLA-DR by colonic CD $4+\mathrm{T}$ cells (3.2 vs. $0.4 \%$, respectively; $P<0.001$ ). However, levels of CD69 and HLA-DR expression did not differ between HAART-treated and HIV-negative controls on sigmoid CD4 + (CD69, 40.7 vs. $54.7 \% ; P=0.27$; HLA-DR, 3.2 vs. $4.4 \% ; P=0.57$ ) or $\mathrm{CD} 8+\mathrm{T}$ cells (CD69, 35.4 vs. $24.8 \%$; $P=0.25$ ). Colonic HIV proviral DNA levels were correlated with increased mucosal CD8 + T-cell immune activation, as reflected by CD69 + expression $(r=0.7$; $P=0.02$; Figure 2c). 

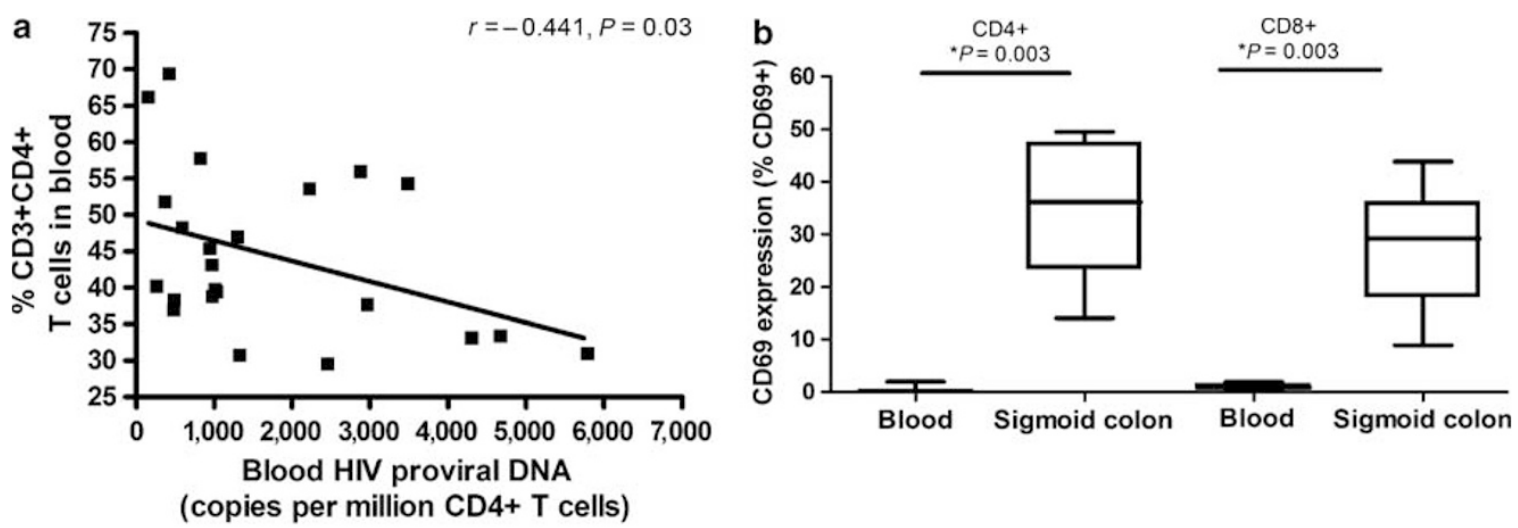

(copies per million CD4+ T cells)

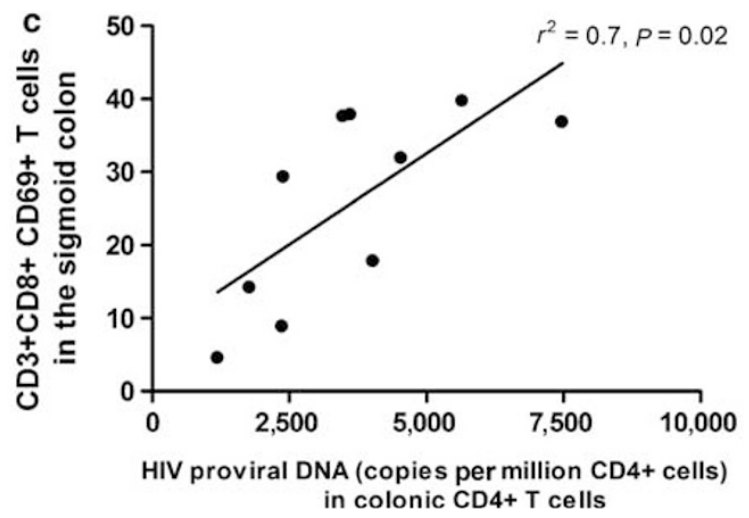

Figure 2 Immune activation and HIV proviral DNA in blood and sigmoid colon. (a) Blood proviral HIV DNA was negatively correlated with the CD4+ T-cell proportion in the blood of HIV-infected individuals. (b) Expression of activation marker CD69+ on colonic and blood CD4+ and CD8+ T cells. (c) A positive correlation was observed between \%CD8+CD69+ expression and HIV proviral DNA in the sigmoid colon. HIV, Human Immunodeficiency Virus.

\section{HIV-specific T-cell responses}

HIV Gag-specific T-cell responses were measured in blood and the sigmoid colon of 16 out of 23 (70\%) participants. Interferon$\gamma$ and/or tumor necrosis factor- $\alpha$ immune responses against HIV Gag were detected in the colon of seven out of $16(44 \%)$ and in the blood of 10 out of $16(63 \%)$ patients (Figure 3a). There was no association between CD4/CD8 + T-cell ratio and the presence or absence of HIV Gag-specific T-cell responses in either compartment (sigmoid, 50 vs. 55\%; $P=0.46$; blood, 45 vs. $48 \%$; $P=0.5$ ). Likewise, no association was seen between CD4/CD8 + T-cell ratio and the strength of HIV-specific T-cell responses, using either interferon- $\gamma$ or tumor necrosis factor- $\alpha$ as a functional output. Neither CD $4+$ nor CD8 + T-cell responses in the blood or the sigmoid colon were associated with HIV proviral DNA levels at their respective sites $(P>0.4$ for both, Figure 3b).

\section{Regulatory T-cell populations in blood and the sigmoid colon}

Regulatory T cells were defined as CD4 + T cells expressing both FoxP3 and CD25 (representative dot plot; Figure 4a), and were significantly increased in sigmoid colon relative to blood in HIVinfected participants (mean, 1.2 vs. $0.2 \%$ of CD $4+\mathrm{T}$ cells; paired $t$-test $P=0.002$; Figure 4b) and HIV-negative controls (data not shown). Regulatory T-cell proportions were much lower in the blood of HIV-infected individuals on long-term HAART than in HIV-uninfected controls (mean, \%CD4 + CD25 + FoxP3 +,
0.18 vs. $1.12 \%$, respectively; $P=0.009$ ), but in contrast to previous reports ${ }^{27}$ did not differ in the sigmoid colon ( 0.56 vs. $0.88 \%$, respectively; $P=0.49$ ). T-regulatory populations in the sigmoid colon did not correlate with mucosal immune activation, although in blood there was a positive correlation with CD4 + T-cell expression of HLA-DR $(r=0.57 ; P=0.02)$. There were no association in either compartment between regulatory T-cell populations and duration of therapy or time to initiation of therapy $(P>0.1$ for both).

\section{DISCUSSION}

The early damage inflicted by HIV at the level of the host gastrointestinal mucosa may set the stage for subsequent systemic inflammation and progressive immune dysfunction. $5,28,29$ Despite the dramatic effects of HAART on blood CD4 + T-cell counts and host survival, it has been unclear to what degree antiretroviral therapy is able to correct these mucosal immune defects, as significantly reduced CD $4 / 8+$ T-cell ratios persist despite effective therapy. ${ }^{13,30}$ It is therefore encouraging that, in this selected cohort of HIV-infected men on long-term suppressive HAART, CD4 + T-cell reconstitution in the sigmoid colon actually exceeded that observed in the blood, with CD4+ T-cell proportions in the colonic mucosa of HAART-treated men approximating those of HIV-uninfected controls. Furthermore, similar degrees of reconstitution were observed in the proportion of colonic CD4 + T-cells expressing the HIV coreceptor and 
a

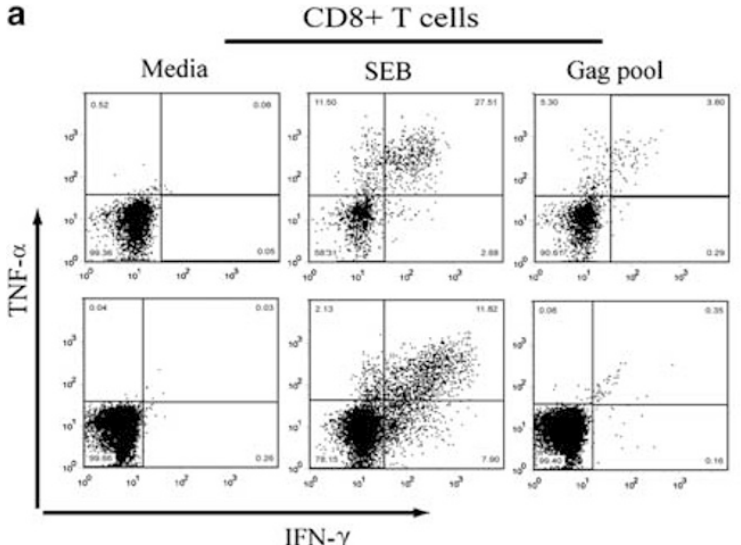

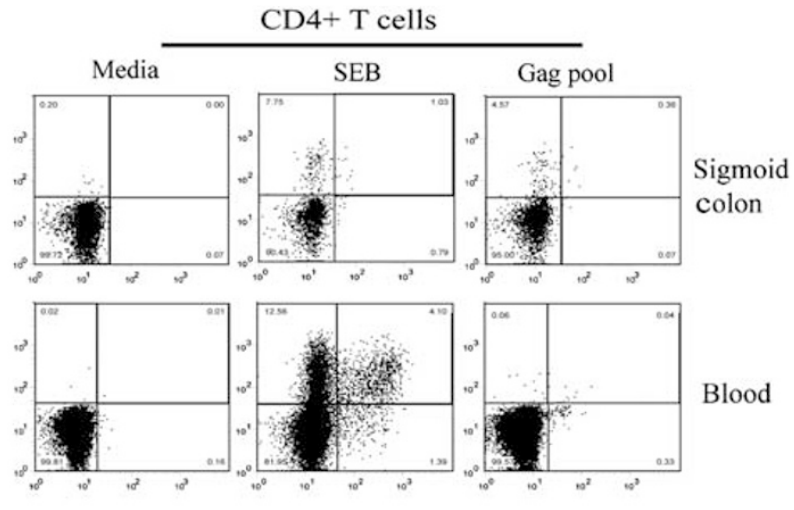

b

NS $(P>0.4)$

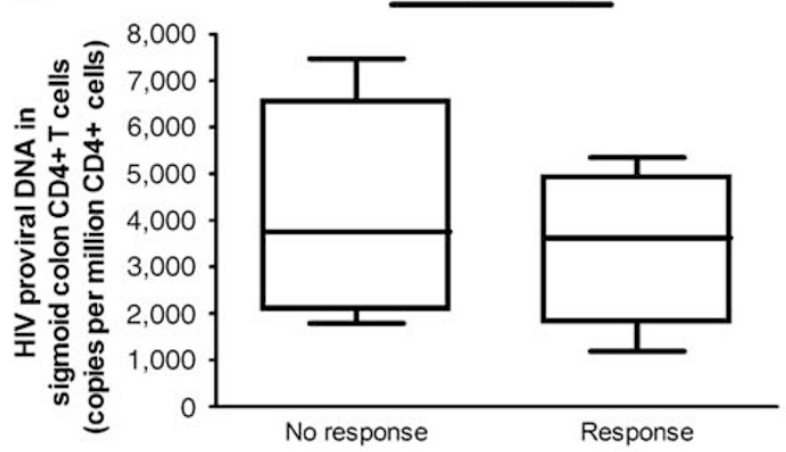

Gag-specific CD8+ T-cell immune response

Figure 3 HIV Gag-specific T-cell responses in blood and sigmoid colon. (a) Gag-specific CD4+ and CD8+ T-cell immune responses in cells isolated from the sigmoid colon (representative dot plots; top panels) and blood (representative dot plots; lower panels). (b) No association was seen between sigmoid HIV proviral load and Gag-specific HIV T-cell immune responses in the sigmoid colon. HIV, Human Immunodeficiency Virus.

activation marker CCR5. However, although increased CD4+ T-cell proportions in the gastrointestinal mucosa have been linked to increased expression of genes involved in growth/ repair and reduction in the expression of genes associated with inflammation, cell activation, and ultimately slower disease progression, ${ }^{30}$ the clinical implications of our findings are not known.

The highly selected participants in this study had unusually complete virus suppression on HAART, but results from this subgroup clearly demonstrate that normalization of colonic CD4 + T-cell proportions is possible on HAART. Prior studies have found persistent depletion of CD4 + T cells in the rectosigmoid colon, particularly CD4 + cells expressing CCR5, after 3 years or more of HAART. ${ }^{13}$ Our positive findings might relate to the increased duration and perhaps more complete viral suppression in our study population. However, a very recent study found that GALT immune reconstitution was incomplete in the terminal ileum (lower small intestine) of a similar highly suppressed patient population. ${ }^{14}$ Although small intestinal biopsies were not collected in our study, this suggests the possibility that there may be differential immune reconstitution in the mucosa of the large vs. small intestine after long-term viral suppression on HAART. Confirmation of this hypothesis will require further studies with sampling of both the large and the small intestinal mucosa.
HIV preferentially infects and replicates in activated CD4+ T cells. This may underlie the viral predilection for the gastrointestinal mucosa, where the number of activated, proliferating CD $4+$ T cells is high. ${ }^{13}$ Levels of immune activation may be elevated in both blood and colonic mucosa of HIV-infected, therapy-naive individuals, based on expression of HLA-DR. ${ }^{13}$ Immune activation in the colon of our participants on long-term HAART, as assayed by expression of HLA-DR and the activation marker CD69, was similar to uninfected controls. Despite this, the size of HIV proviral reservoir in the colon was correlated with the degree of CD8 + T-cell immune activation, implying that even a "normalized" level of immune activation in the gut mucosa may be sufficient to support low-level virus replication despite HAART.

In HAART-naive individuals, HIV Gag-specific CD8 + T-cell responses in blood have been associated with reduced viremia and slower disease progression. ${ }^{24} \mathrm{HIV}$-specific CD $8+\mathrm{T}$-cell responses are also detectable at mucosal sites, including the gut, ${ }^{31}$ cervix, ${ }^{32,33}$ and semen, ${ }^{34,35}$ although their clinical significance at these mucosal sites is less clear. In our highly suppressed participants, Gag-specific CD8 + (and to a lesser degree CD4 + ) $\mathrm{T}$-cell immune responses were often detectable in the blood and the colonic mucosa, but neither their presence nor their magnitude was associated with the degree of CD4 + T-cell reconstitution 
a
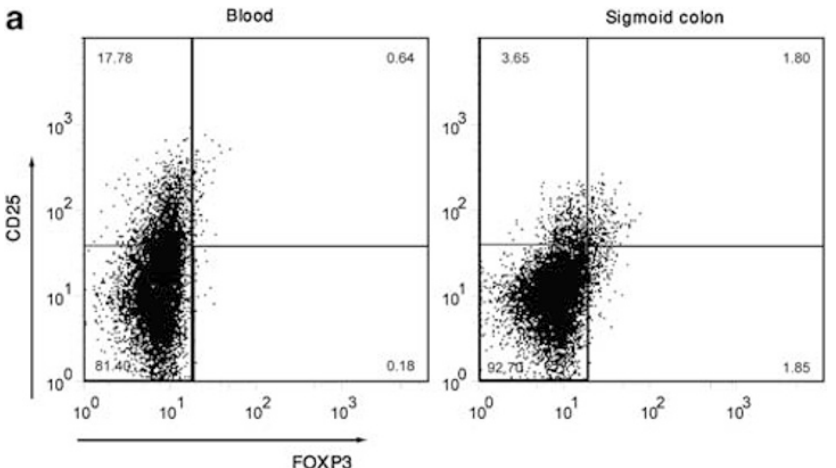

b

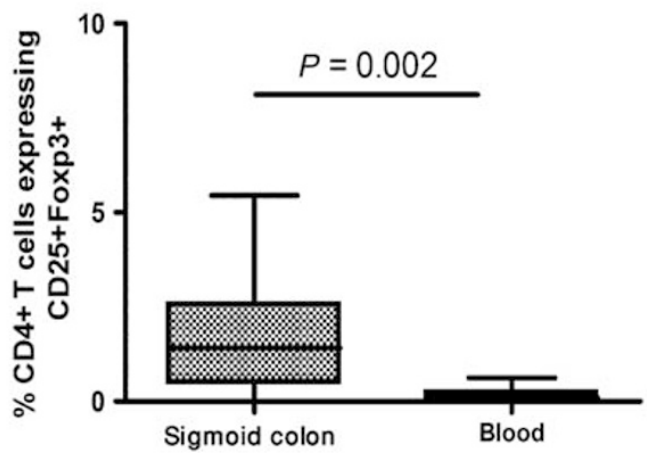

Figure 4 Proportions of regulatory T cells in blood and sigmoid colon. (a) Representative dot plots comparing CD4 $+\mathrm{T}$ cells coexpressing CD25+FoxP3 + in blood (left panel) and sigmoid colon (right panel) in an HIV-infected individual on long-term suppressive HAART.

(b) A significantly elevated proportion of CD4 + T cells coexpressing CD25+FoxP3 in the sigmoid colon compared to blood of

HIV-infected men on long-term suppressive HAART. HAART, highly active antiretroviral therapy; HIV, Human Immunodeficiency Virus.

or the HIV latent proviral DNA load at either site. However, we examined only a limited repertoire of T-cell immune functions (production of tumor necrosis factor- $\alpha$ and interferon- $\gamma$ ), and the immune techniques used did not permit us to determine the clonal diversity of the HIV-specific T-cell immune responses observed. Specifically, responses may have represented the preservation or reconstitution of a clonally diverse T-cell population, or the re-expansion of an oligo-clonal response from a small reservoir of memory effector $\mathrm{T}$ cells. In addition, cell numbers did not permit detailed mapping of the antigen specificity of the responses seen, a factor that may impact host immune control of virus. Nonetheless, as blood HIV-specific CD8 + responses wane on HAART, in the absence of antigenic stimulation, ${ }^{36}$ the detection of responses in our participants implies that there is ongoing low-level viral replication in blood and sigmoid colon despite long-term suppressive therapy.

In summary, levels of CD4 + T-cell reconstitution in the sigmoid colon of this selected group on highly suppressive antiretroviral therapy actually exceeded those observed in blood, with $\mathrm{CD} 4 / \mathrm{CD} 8$ + ratios comparable to uninfected controls. However, the persistence of HIV-specific CD8 + T-cell responses in both the blood and the recto-sigmoid suggests that there may be ongoing low-level HIV replication in the gastrointestinal mucosa despite prolonged undetectable viremia. Although complete normalization of CD4 + T-cell proportions in the sigmoid colon can be achieved on long-term suppressive HAART, the clinical importance of such normalization remains unclear and will be an important area for further study.

\section{METHODS}

Study participants. HIV-infected and -uninfected controls were recruited through the Canadian Immunodeficiency Research Collaborative at the Maple Leaf Medical Clinic in Toronto, Canada. All HIV-infected participants had been on uninterrupted HAART for at least 48 months with an undetectable blood HIV viral load ( $<50$ copies/ $\mathrm{ml}$ ). All subjects provided informed, written consent. The study protocol was approved by Research Ethics Boards at the University Health Network, Toronto, the University of Toronto, and St Michael's Hospital, Toronto.

Sampling protocol. Blood was obtained by venipuncture and collected into Acid Citrate Dextran solution A (BD Bioscience, La Jolla, CA). Plasma viremia was determined using the Versant HIV-1 RNA 3.0 assay (bDNA; Bayer Diagnostics, Puteaux Cedex, France). Peripheral blood mononuclear cells were isolated by density centrifugation as previously described..$^{34}$ Between 20 and 25 biopsies were obtained from the recto-sigmoid, approximately $25-30 \mathrm{~cm}$ from the anal verge. Biopsies were immediately placed into RPMI containing $100 \mathrm{U} \mathrm{ml}^{-1}$ penicillin, $100 \mu \mathrm{g} \mathrm{ml}^{-1}$ streptomycin, and $1 \times$ GlutaMAX-1 (Invitrogen, Carlsbad, CA). Colonic mucosal mononuclear cells were isolated by digestion with 0.5-1.0 $\mathrm{mg} \mathrm{ml}^{-1}$ of Collagenase type II (Clostridiopeptidase A; SigmaAldrich, St Louis, MI) for $30 \mathrm{~min}$ on a stirring heating block. The digested cell suspension was filtered through a $100-\mu \mathrm{m}$ filter and enumerated.

Evaluating HIV-specific immune responses ex vivo. HIV Gagspecific CD $4+$ and CD $8+T$ cell-specific immune responses were measured in blood and the sigmoid colon, using modifications of a previously described protocol. ${ }^{34}$ One million colonic mucosal mononuclear cells and peripheral blood mononuclear cells were incubated in media with $2 \%$ dimethyl sulfoxide (negative control), or stimulated using Staphlococcus enterotoxin-B (SEB, positive control; $3 \mu \mathrm{g} \mathrm{ml}^{-1}$ ) or Gag Clade-B peptide pool $\left(1 \mu \mathrm{g} \mathrm{ml}^{-1}\right.$ per peptide $)$ for $1 \mathrm{~h}$ at $37^{\circ} \mathrm{C}$ and $5 \% \mathrm{CO}_{2}$. No costimulatory molecules were used. Clade-B Gag pool was made up of 122 15-mer overlapping peptides encompassing the entire Gag gene. Brefeldin A $\left(1 \mu \mathrm{g} \mathrm{ml}^{-1}\right)$ was added, and samples were incubated for another $5 \mathrm{~h}$ at $37^{\circ} \mathrm{C}$ and $5 \% \mathrm{CO}_{2}$. Samples were washed with $1 \%$ fetal bovine serum-phosphate-buffered saline, permeabilized, and stained with fluorochrome-labeled monoclonal antibodies specific for CD8, CD3, tumor necrosis factor- $\alpha$, and interferon- $\gamma$ (Becton Dickinson Immunocytometry Systems, San Jose, CA). Samples were acquired using a FACSCalibur flow cytometer (BD Systems) and data analysis performed using Flow Jo analytical software version 7.2.2 (Treestar, Ashland, OR). A positive response was defined as background-corrected cytokine production in the HIV Gag well exceeding $0.05 \%$ of gated cells and background levels of cytokine production by at least twofold.

Measurement of proviral HIV DNA. CD4 $+\mathrm{T}$ cells were isolated from peripheral blood mononuclear cells using a column-based cell separation technique (StemCell Technologies, Vancouver, BC, Canada) as previously described. ${ }^{37}$ To determine the proviral HIV DNA copies per million CD4 + T cells, genomic DNA was isolated using the Puregene DNA isolation kit according to the manufacturer's specifications (Gentra, Minneapolis, MN). One microgram of DNA was then used as template for real-time PCR in an iCycler (Bio-Rad, Hercules, CA). The amplification reaction was carried out in triplicate using $0.5 \mu \mathrm{M}$ primers, $0.2 \mu \mathrm{M}$ fluorescent probe, $0.8 \mathrm{mM}$ dNTPs, $5 \mathrm{mM} \mathrm{MgCl}_{2}$, and $2.5 \mathrm{U}$ Platinum Taq Polymerase (Invitrogen) in $50 \mu \mathrm{l}$ total volume. The following primers were used: 5' -GGTCTCTCTGGTTAGACCAGAT-3' (5' primer) and $5^{\prime}$-CTGCTAGAGATTTTCCACACTG-3' (3' primer) along with the fluorescent probe $5^{\prime}$-6FAM-AGTAGTGTGTGCCCGTCTGTTTAMRA-3'. PCR conditions consisted of a denaturation step at 
$95^{\circ} \mathrm{C}$ for $3 \mathrm{~min}$ followed by 45 cycles of $15 \mathrm{~s}$ at $95^{\circ} \mathrm{C}$ and $1 \mathrm{~min}$ at $59^{\circ} \mathrm{C}$. Serially diluted ACH-2 DNA was also subjected to the above PCR to obtain standard curves. Approximately, 200,000 CD8-depleted cells were lysed in $10 \mathrm{mM}$ Tris- $\mathrm{HCl}$ ( $\mathrm{pH} 8$ ) containing $100 \mu \mathrm{g} \mathrm{ml}^{-1}$ proteinase $\mathrm{K}$ (Roche Applied Science, Indianapolis, IN) for $1 \mathrm{~h}$ at $56^{\circ} \mathrm{C}$ followed by heat inactivation of the enzyme. Real-time PCR specific for human $\beta$-actin DNA (Applied Biosystems, Foster City, CA) was carried out on the above cell lysates to determine the exact copy number of cells per $\mu \mathrm{l}$ of cell lysate. Serially diluted ACH-2 DNA was also subjected to the above PCR to obtain standard curves. Finally, real-time PCR specific for HIV DNA was carried out as described above, and the copy number of HIV DNA per $1 \times 10^{6} \mathrm{CD} 4+\mathrm{T}$ cells was calculated based on the results obtained from FACS and PCR experiments.

Statistical analysis. SPSS 14 for Windows XP (SPSS, Chicago, IL) was used for statistical analysis. Intraindividual comparisons of T-cell populations in blood and sigmoid colon were performed using the paired samples $t$-test. The Mantel-Haenszel $\chi^{2}$ test with calculation of likelihood ratios and confidence intervals was used to compare dichotomous variables between study groups, and comparison of means between groups was performed by the Mann-Whitney $U$ non-parametric test. Linear association of continuous variables was assessed using the Spearman's rank correlation coefficient.

\section{ACKNOWLEDGMENTS}

This work was supported in part by the Ontario HIV Treatment Network (P.S., salary award); the CIHR (R.K., HOP-81735 and HET-85518); the Canadian Research Chair Program (R.K., salary support); and an unrestricted research grant from Gilead Pharmaceuticals (M.O., C.K., and T.C.). Study sponsors played no role in study design, collection or analysis of data, interpretation of results, writing of the manuscript, or decision to submit for publication.

\section{DISCLOSURE}

The authors have no competing interests.

\section{Author contributions}

P.S., R.K., M.O., and T.C. designed the study. P.S. and R.K. analyzed the data. D.P., M.L., C.K., and R.H. enrolled the patients. P.S., R.K., and M.O. contributed to writing the paper. P.S., D.C., S.H., L.S., and F.Y. collected data and performed the experiments.

\section{() 2008 Society for Mucosal Immunology}

\section{REFERENCES}

1. UNAIDS/WHO AIDS epidemic update-December 2006.http://www. unaids.org/en/HIV_data/epi2006/ (2006).

2. Douek, D. HIV disease progression: immune activation, microbes, and a leaky gut. Top. HIV Med. 15, 114 (2007)

3. Lim, S.G. et al. Loss of mucosal CD4 lymphocytes is an early feature of HIV infection. Clin. Exp. Immunol. 92, 448 (1993).

4. Mowat, A.M. \& Viney, J.L. The anatomical basis of intestinal immunity. Immunol. Rev. 156, 145 (1997).

5. Brenchley, J.M. et al. CD4+ T cell depletion during all stages of HIV disease occurs predominantly in the gastrointestinal tract. J. Exp. Med. 200, 749 (2004).

6. Mehandru, S. et al. Primary HIV-1 infection is associated with preferential depletion of CD4+ T lymphocytes from effector sites in the gastrointestinal tract. J. Exp. Med. 200, 761 (2004).

7. Mattapallil, J.J. et al. Massive infection and loss of memory CD4+ T cells in multiple tissues during acute SIV infection. Nature 434, 1093 (2005).

8. $\mathrm{Li}, \mathrm{Q}$. et al. Peak SIV replication in resting memory CD4+ T cells depletes gut lamina propria CD4+ T cells. Nature 434, 1148 (2005).

9. Veazey, R.S. et al. Gastrointestinal tract as a major site of CD4+ T cell depletion and viral replication in SIV infection. Science 280, 427 (1998).

10. Brenchley, J.M., Price, D.A. \& Douek, D.C. HIV disease: fallout from a mucosal catastrophe? Nat. Immunol. 7, 235 (2006).

11. Lederman, M.M. \& Valdez, H. Immune restoration with antiretroviral therapies: implications for clinical management. JAMA 284, 223 (2000).
12. Guadalupe, M. et al. Severe CD4+ T-cell depletion in gut lymphoid tissue during primary human immunodeficiency virus type 1 infection and substantial delay in restoration following highly active antiretroviral therapy. J. Virol. 77, 11708 (2003).

13. Mehandru, S. et al. Lack of mucosal immune reconstitution during prolonged treatment of acute and early HIV-1 infection. PLOS Med. 3 , e484 (2006).

14. Chun, T.W. et al. Persistence of HIV in Gut-associated lymphoid tissue despite long-term antiretroviral therapy. J. Infect. Dis. 197, 714 (2008).

15. Dandekar, S. Pathogenesis of HIV in the gastrointestinal tract. Curr. HIVIAIDS Rep. 4, 10 (2007).

16. Sankaran, S. et al. Gut mucosal T cell responses and gene expression correlate with protection against disease in long-term HIV-1-infected nonprogressors. Proc. Natl. Acad. Sci. USA 102, 9860 (2005).

17. Brenchley, J.M. \& Douek, D.C. HIV infection and the gastrointestinal immune system. Mucosal. Immunol. 1, 23 (2007).

18. Giorgi, J.V. et al. Shorter survival in advanced human immunodeficiency virus type 1 infection is more closely associated with T lymphocyte activation than with plasma virus burden or virus chemokine coreceptor usage. J. Infect. Dis. 179, 859 (1999).

19. Hazenberg, M.D. et al. Persistent immune activation in HIV-1 infection is associated with progression to AIDS. AIDS 17, 1881 (2003).

20. Sodora, D.L. \& Silvestri, G. Immune activation and AIDS pathogenesis. AIDS 22, 439 (2008).

21. Kinter, A.L. et al. CD25(+)CD4(+) regulatory T cells from the peripheral blood of asymptomatic HIV-infected individuals regulate CD4(+) and CD8(+) HIV-specific T cell immune responses in vitro and are associated with favorable clinical markers of disease status. J. Exp. Med. 200, 331 (2004).

22. Belkaid, Y. \& Rouse, B.T. Natural regulatory T cells in infectious disease. Nat. Immunol. 6, 353 (2005).

23. Jin, X. et al. Dramatic rise in plasma viremia after CD8(+) $T$ cell depletion in simian immunodeficiency virus-infected macaques. J. Exp. Med. 189, 991 (1999).

24. Kiepiela, P. et al. CD8+ T-cell responses to different HIV proteins have discordant associations with viral load. Nat. Med. 13, 46 (2007).

25. Pontesilli, O. et al. Longitudinal analysis of human immunodeficiency virus type 1-specific cytotoxic T lymphocyte responses: a predominant gag-specific response is associated with nonprogressive infection. J. Infect. Dis. 178, 1008 (1998).

26. Shacklett, B.L. et al. Trafficking of human immunodeficiency virus type 1-specific CD8(+) T cells to Gut-associated lymphoid tissue during chronic infection. J. Virol. 77, 5621 (2003).

27. Epple, H.J. et al. Mucosal but not peripheral FOXP3+ regulatory T cells are highly increased in untreated HIV infection and normalize after suppressive HAART. Blood 108, 3072 (2006).

28. Haase, A.T. Perils at mucosal front lines for HIV and SIV and their hosts. Nat. Rev. Immunol. 5, 783 (2005).

29. George, M.D. et al. High-throughput gene expression profiling indicates dysregulation of intestinal cell cycle mediators and growth factors during primary simian immunodeficiency virus infection. Virology 312, 84 (2003).

30. Guadalupe, M. et al. Viral suppression and immune restoration in the gastrointestinal mucosa of human immunodeficiency virus type 1-infected patients initiating therapy during primary or chronic infection. J. Virol. 80, 8236 (2006).

31. Shacklett, B.L. et al. Characterization of HIV-1-specific cytotoxic T lymphocytes expressing the mucosal lymphocyte integrin CD103 in rectal and duodenal lymphoid tissue of HIV-1-infected subjects. Virology $\mathbf{2 7 0}$, 317 (2000).

32. Shacklett, B.L. et al. Quantification of HIV-1-specific T-cell responses at the mucosal cervicovaginal surface. AIDS 14, 1911 (2000).

33. Kaul, R. et al. Quantitative ex vivo analysis of functional virus-specific CD8T lymphocytes in the blood and genital tract of HIV-infected women. AIDS 17, 1139 (2003).

34. Sheth, P.M. et al. HIV-specific CD8+ lymphocytes in semen are not associated with reduced HIV shedding. J. Immunol. 175, 4789 (2005).

35. Quayle, A.J. et al. Detection of HIV-1-specific CTLs in the semen of HIV-infected individuals. J. Immunol. 161, 4406 (1998).

36. Kostense, S. et al. High viral burden in the presence of major HIV-specific CD8(+) T cell expansions: evidence for impaired CTL effector function. Eur. J. Immunol. 31, 677 (2001).

37. Chun, T.W. et al. Induction of HIV-1 replication in latently infected CD4+ T cells using a combination of cytokines. J. Exp. Med. 188, 83 (1998). 